\title{
EFEITOS DE PRODUTOS ANABOLIZANTES SOBRE A QUALIDADE DO SÊMEN DE TOUROS
}

\author{
EFFECTS OF ANABOLIC PRODUCTS ON BULL SEMEN QUALITY
}

\author{
Cláudio Alves Pimentel ${ }^{1}$ João Carlos Deschamps ${ }^{2}$
}

\section{RESUMO}

Com a finalidade de se avaliar o efeito de esteróides anabolizantes sobre a qualidade do sêmen de touros, foram testados 3 tratamentos em 9 touros adultos (idades de 3 a 5 anos). Os grupos I e II receberam implantes subcutâneos na base da orelha, sendo implantados no grupo I, $36 \mathrm{mg}$ de zeranol e no grupo II $24 \mathrm{mg}$ de dietiletilbestrol (DES), enquanto o grupo III permaneceu como testemunha. Foram realizadas 31 coletas de sêmen semanais, tendo sido a primeira, uma semana antes do implante. Não se verificaram alterações em nenhuma das características do sêmen devido aos tratamentos. Os três grupos tiveram ganhos de peso similares. Conclui-se que, nas condiçбes em que o experimento foi realizado, não houve efeito dos anabolizantes na qualidade do sêmen. Caso tenham ocorrido alteraçóes genitais mais discretas, essas foram subclínicas ou não foram detectadas devido ao numero de unidades experimentais por tratamento.

Palavras-chave: touro, sêmen, anabolizantes, hormônios, zeranol.

\section{SUMMARY}

In order to evaluate the effect of anabolic steroids on the semen quality of bulls, 3 treatments were tested in 9 adult bulls ( 3 to 5 years old). Groups I and II received

subcutaneous implants in the base of the ear while group III remained as control. Bulls from group I were treated with $36 \mathrm{mg}$ of Zeranol and group II $24 \mathrm{mg}$ of diethylethylbestrol (DES). A total of 31 weekly semen collections were performed, being the first, one week before the implant. No changes on semen characteristics were observed due to treatments. The 3 groups had similar weight gain. It was concluded that, in the conditions of this experiment, there was no effect of the anabolic products on semen quality. In case that some mild genital changes had ocurred, they were subclinical or were not detected due to the number of experimental units per treatment.

Key words: bull, semen, anabolics, hormones, zeranol.

\section{INTRODUÇÃO}

O zeranol, uma lactona do ácido resorcílico, é um estimulante de crescimento que foi descoberto como resultado de pesquisas de STOB et al. (1962). O referido autor, isolou, a partir de milho infectado com um fungo classificado como Giberella zeae, um composto que parecia ser o responsável pelo aparecimento de síndrome estrogênica (hipertrofia vulvar, prolapso vaginal e aumento da glândula mamária em fêmeas e edema de prepacio e glândula mamária em machos) em suínos tratados com milho infectado com o fungo acima citado. A estrutura química desta substância, que possui, ainda atividade estimulante do crescimen-

\footnotetext{
${ }^{1}$ Médico Veterinário, Professor Titular, Departamento de Patologia Animal, Faculdade de Veterinária, Universidade Federal de Pelotas (UFPel). 96010 900 Pelotas, RS. Autor para correspondéncia.

${ }^{2}$ Médico Veterinário, Professor Adjunto, Departamento de Patologia Animal, Faculdade de Veterinária, UFPel.
}

Recebido para publicagăo em 05.07.94. Aprovado en 11.01.95 
to, foi elucidada por URRY et al. (1966) passando a set conhecida pelo nome trivial de Zearalenona. Um derivado desta substância original tem sido produzido em escala comercial e usado como agente anabolizante (Ralgro), para promover aumento no crescimento e conversão alimentar, em bovinos, em certos países. Existem evidências de que estes derivados da Zearalenoma, interagem com os receptores citoplasmáticos do estrogênio, determinando muitas das mesmas características biológicas e respostas bioquímicas provocadas pelo estrogênio natural, também conhecido pelo nome de estradiol (KATZENELLENBOGEN et al., 1979).

JUNIEWICZ et al. (1981) demonstraram que o Zeranol determina uma redução no peso testicular e uma inibição do processo espermatogênico em touros, quando administrado antes do aparecimento da puberdade. Além disso, DESCHAMPS et al. (1987b) demonstraram lesð̃es estruturais irreversíveis no epidídimo, testículo e glândulas anexas, em touros jovens, implantados antes da puberdade. Entretanto, uma vez o touro tendo atingido a maturidade sexual, o tratamento anabolizante não afeta a estrutura histológica da genitália.

Com o objetivo de testar o efeito de substâncias anabolizantes sobre a qualidade do sêmen de touros, foi delineado o presente experimento, tomando como referencia, o uso de Dietiletilbestrol (DES) ${ }^{2}$ e Zeranol ${ }^{b}$.

\section{MATERIAL E MÉTODOS}

Foram utilizados nove touros da raça Charolesa, com idade variando entre três e cinco anos, tendo sido os animais divididos, ao acaso, em três grupos de três animais cada um. Cada um dos grupos foi submetido a um diferente tratamento. $O$ grupo I recebeu um implante de $36 \mathrm{mg}$ de Zeranol, na base da orelha, enquanto que o grupo II recebeu, também através de implante na base da orelha, $24 \mathrm{mg}$ de dietiletilbestrol (DES), permanecendo o grupo III como controle.

Foram realizadas coletas de sêmen dos animais em questão, em intervalos semanais e, eventualmente, quinzenais, totalizando 31 coletas realizadas, tendo sido a primeira, uma semana antes do implante. $O$ exame do sêmen foi realizado segundo DESCHAMPS \& PIMENTEL (1979). Os caracteres avaliados no exame de sêmen compreendiam volume, turbilhonamento, motilidade, vigor, concentração e morfologia espermática (gota citoplasmática procimal, gota citoplasmática distal, anormalidades de cabeça, anormalidades de peça intermediária, anormalidades de cauda e defeitos de acrossoma).

$O$ delineamento experimental foi completamente ao acaso e a análise de variânça foi conduzida através de um arranjo de tratamentos em parcelas subdivididas, sendo, as parcelas, os tratamentos (Zeranol, DES e controle), e as subparcelas, os períodos de coleta de sêmen, em dias. $\mathrm{O}$ comportamento das diferentes variáveis, em função do tempo, foi submetida à uma análise de regressão (STEEL \& TORRIE, 1960).

\section{RESULTADOS}

As características do sêmen consideradas no presente experimento (Tabela 1), não sofreram efeitos devido aos tratamentos (Zeranol, DES e Controle), porém, aquelas que variaram em função do tempo, foram representadas graficamente como um anico grupo.

No entanto, aquelas que variaram em função do tempo (Figura 1), foram representadas graficamente como um único grupo.

- o peso corporal $\left(R^{2}=84,48 \%, P<0,01\right.$, Figura 1a);

- a concentracão do sêmen $\left(R^{2}=48,73 \%, P<0,09\right.$, Figura 1b);

- a presença de espermatozóides normais $\left(R^{2}=55,66 \%\right.$, $P<0,06$, Figura 1c);

- anormalidades de cabeça $\left(R^{2}=35,51 \%, P<0,02\right.$, Figura 1d) $\mathrm{e}$

- anormalidades de cauda dos espermatozoides $\left(R^{2}=\right.$ $24,95 \%, P<0,01$, Figura 1e). Essas variáveis, sofreram mudanças em função do tempo, sem qualquer interação com os tratamentos das parcelas. A motilidade, no entanto, não se apresentou alterada nos diferentes tratamentos, nem sofreu variação, em função do tempo. Houve um ganho de peso significativo, nos três grupos até 100 dias após o início dos tratamentos (Figura 1a).

Tabela 1. Médias* das diferentes variáveis para cada tratamento.

\begin{tabular}{lccc}
\hline Variáveis & Grupo I & Grupo II & Grupo III \\
\hline $\begin{array}{l}\text { Concentração } \\
\left(\times 10^{3} \text { esp } / \mathrm{mm}^{3}\right)\end{array}$ & 832,2 & 831,8 & 689,3 \\
Motilidade (\%) & 69,3 & 69,6 & 67,4 \\
$\begin{array}{l}\text { Espermatozoides } \\
\text { Normais }(\%)\end{array}$ & 94,6 & 94,6 & 92,9 \\
$\begin{array}{l}\text { Anormalidades } \\
\text { de cabeça (\%) }\end{array}$ & 2,7 & 3,8 & 3,4 \\
$\begin{array}{l}\text { Anormalidades } \\
\text { de cauda }(\%)\end{array}$ & 3,0 & 2,5 & 2,6 \\
$\begin{array}{l}\text { Peso } \\
\text { Corporal }(\mathrm{kg})\end{array}$ & 570,3 & 558,8 & 567,0 \\
\hline
\end{tabular}

(*) Obs. Não houve efeito significativo ( $P>0,05)$ entre os tratamentos (parcelas), porém houve efeito das sub-parcelas (não houve interação). 


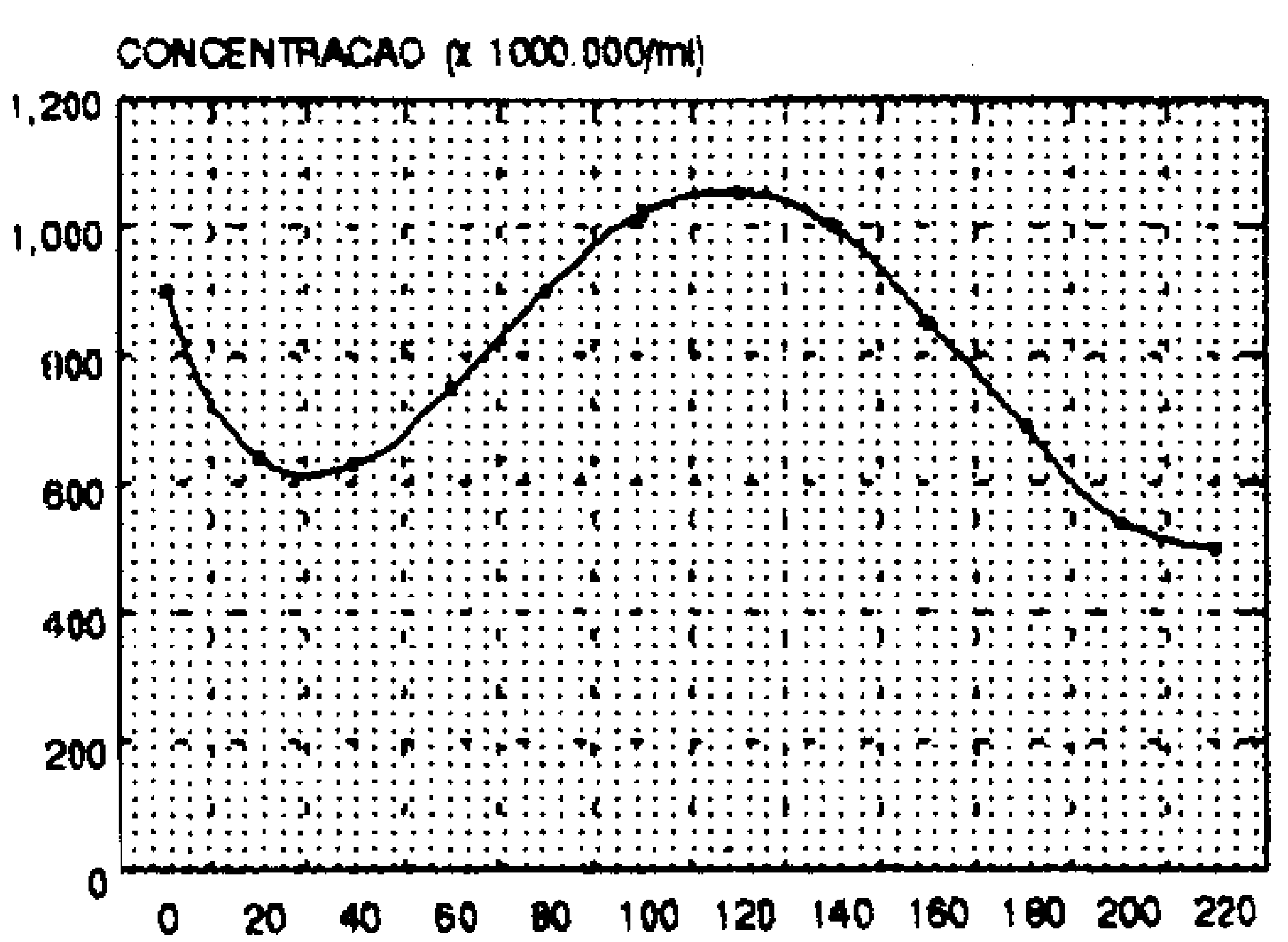

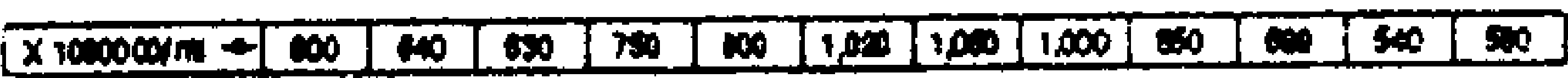

TEMPO (diest

ANOFMAUDADES DE CABECA ( $\%$

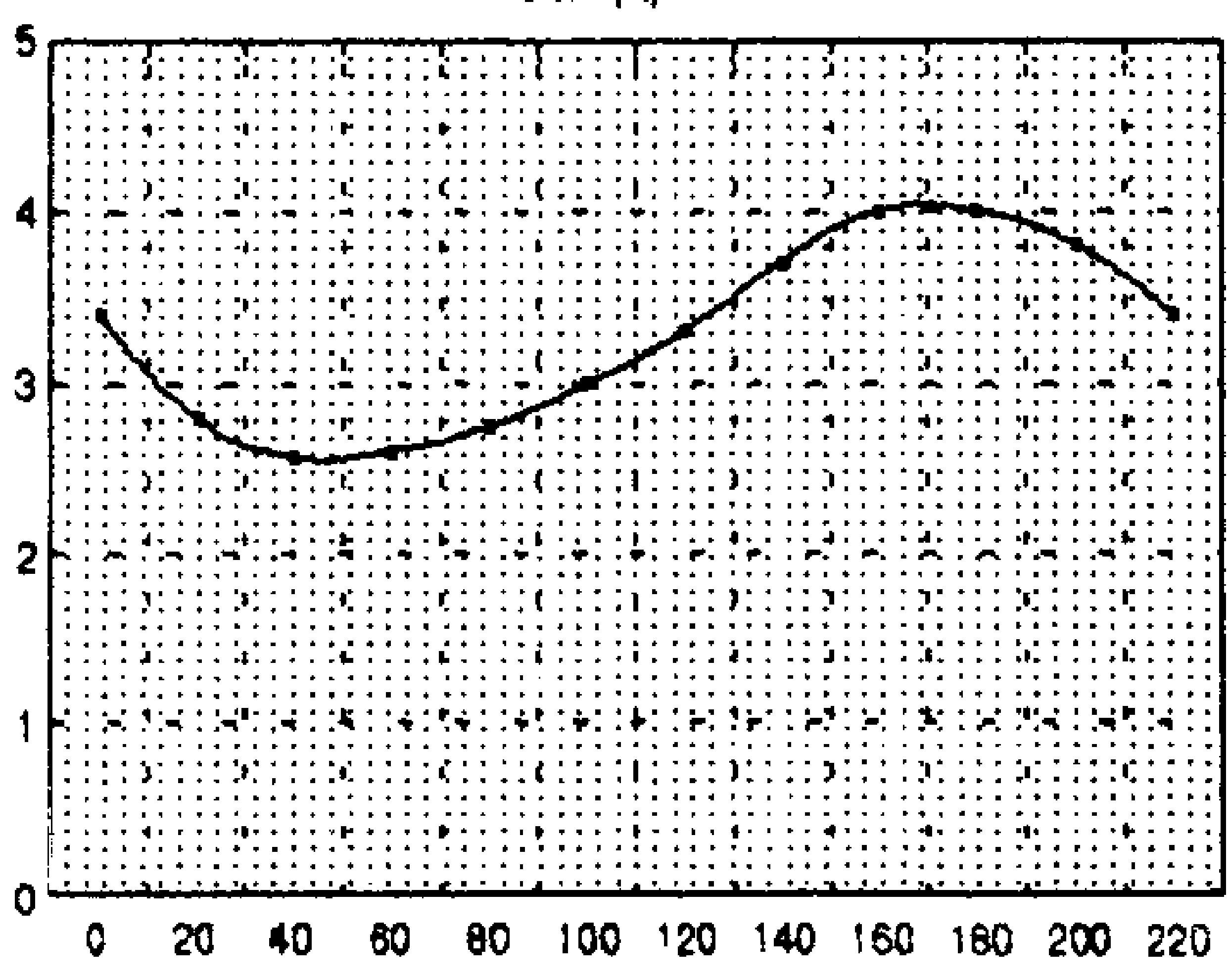

TEMPO (dian

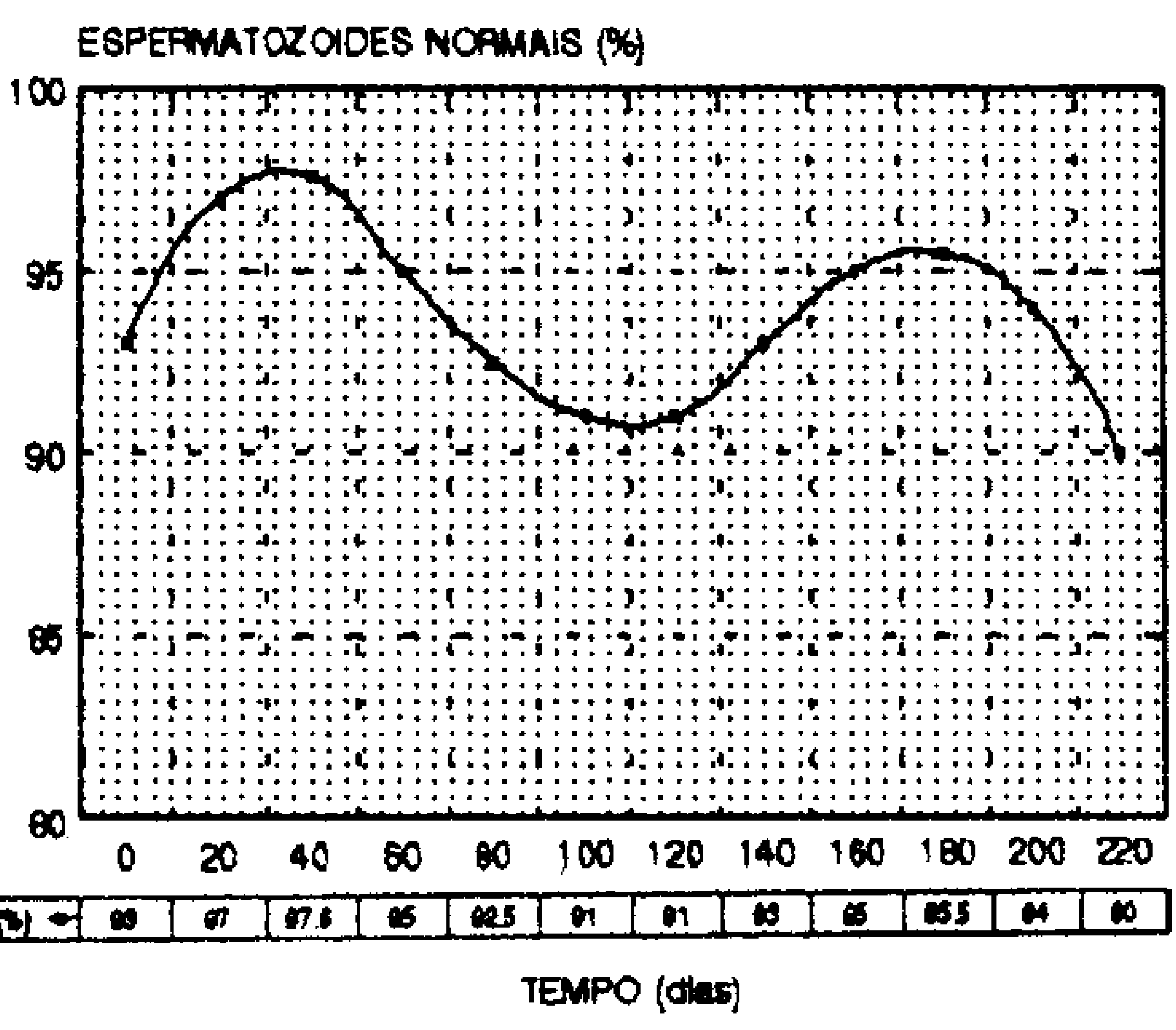

ANOAMAUDADES DE CAUDA (W)

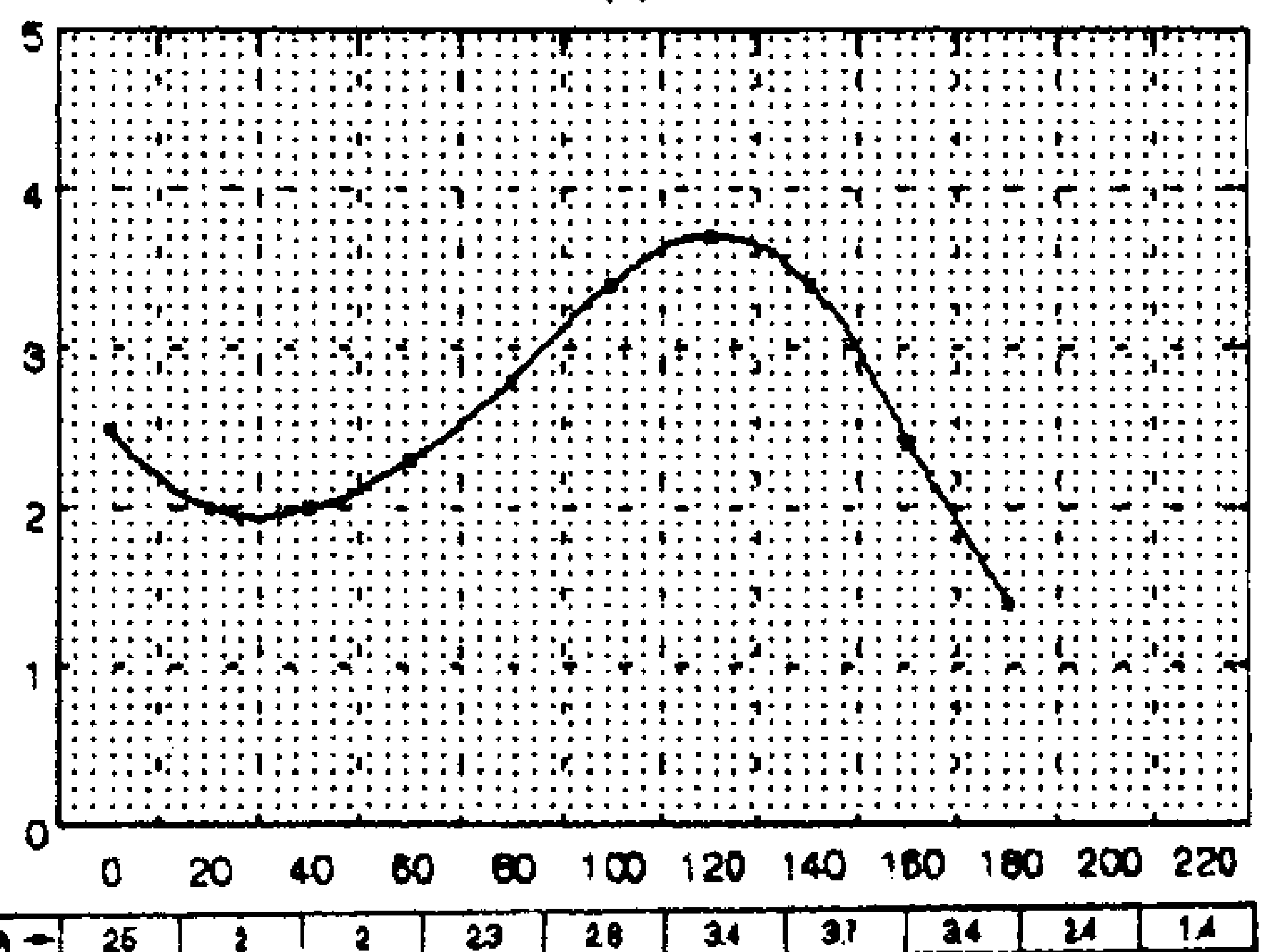

TEMPO (dies)

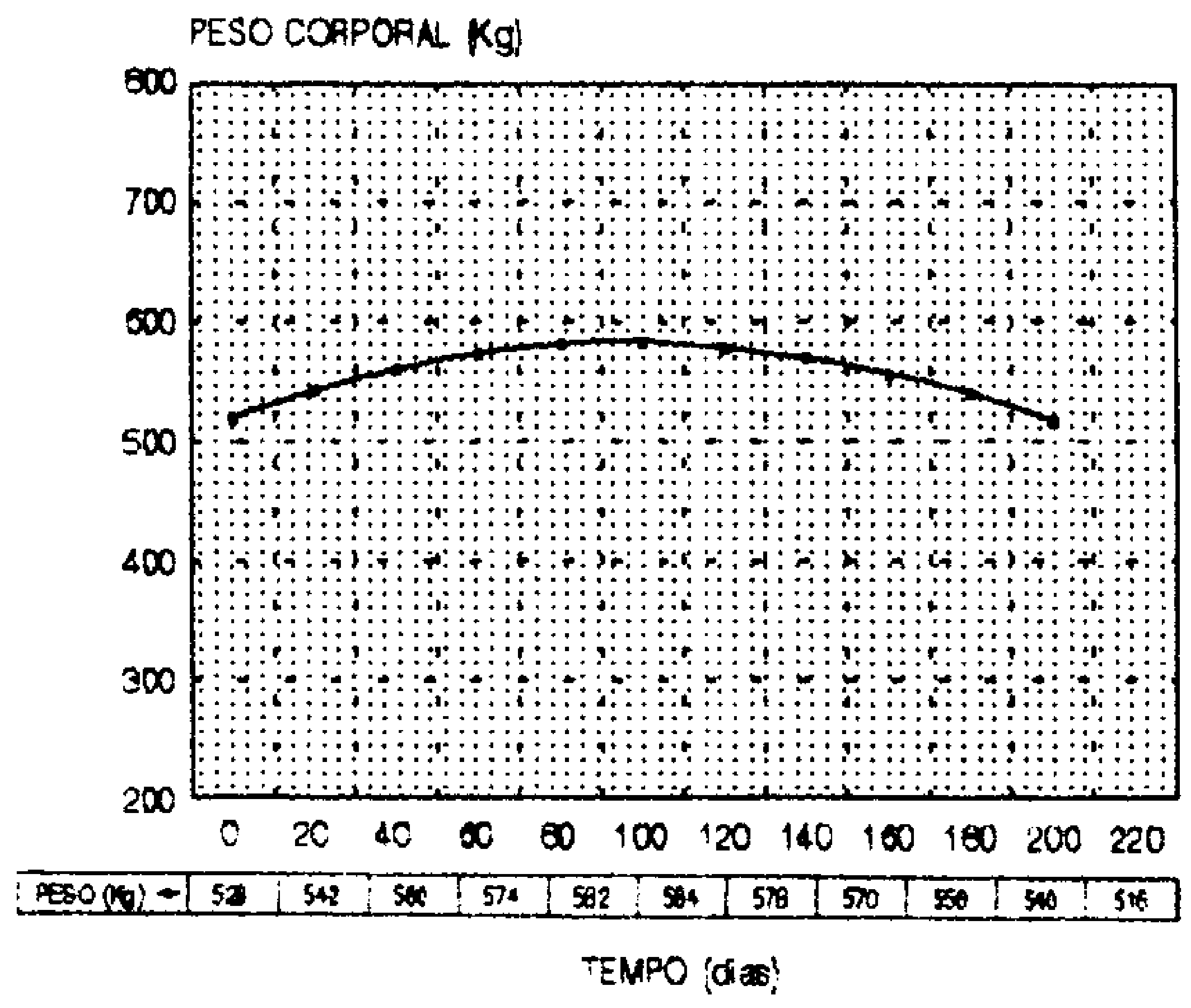

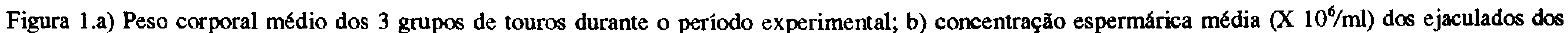
3 grupos de touros durante o período experimental; c) percentagem de espermatozóides normais dos ejaculados dos 3 grupos de touros durante o período experimental; d) anormalidades de cabeça média (\%) dos ejaculados dos 3 grupos de touros durante o período experimental; anormalidades de cauda $(\%)$ média dos ejaculados dos 3 grupos de touros durante o período experimental. 


\section{DISCUSSÃO}

Pela observação dos resultados da análise de variança das diferentes características consideradas, pode se verificar a ausência de efeitos do Zeranol e DES, quando comparados ao grupo controle, sobre a qualidade do sêmen, aceitando-se, como verdadeira, a hipótese de nulidade. Estes resultados são contraditórios ao que seria de esperar, ou seja, deteriorização na qualidade do sêmen sob efeito de substâncias estrogenizantes (CUPPS et al., 1960; GUSTAFSSON, 1966; CUPPS, 1975), tais como queda na concentração, na motilidade e aumento na quantidade de espermatozóides com anormalidade de cauda.

Alterações específicas no espermiograma tem sido registradas por outros autores em decorrência de elevadas concentrações plasmáticas e endógenas de cortisol (CUPPS, 1975) ou exógenas de testosterona (RICHCKIND et al., 1967) e progesterona (MATSUYAMA et al., 1967). DESCHAMPS et al. (1987b) observaram, em touros jovens (pré-puberes) implantados com Zeranol, uma redução do volume, concentração e motilidade do ejaculado associados a um aumento de formas anormais dos espermatozoides ejaculados. Esses autores atribuiram tais efeitos a redução do desenvolvimento testicular dos animais implantados com Zeranol. Neste trabalho, não se verificou variação significativa na circunferência escrotal devido aos implantes (Zeranol e DES), porém isso ocorreu, possivelmente, porque os touros usados eram adultos (DESCHAMPS et al., 1987a).

A inexistência de efeito significativo do Zeranol sobre a qualidade do sêmen em relação ao grupo controle, pode ser atribuido à menor potência estrogênica deste produto, em relação ao Estradiol $(13,6 \%$, segundo KATZENELLENBOGEN et al., 1979). Também tal resultado está de acordo com a observação de JUNIEWICZ et al. (1981), indicando que o efeito prejudicial do Zeranol sobre a função testicular, não se faz notar em animais sexualmente adultos e, possivelmente, as alterações histologicas decorrentes do efeito estrogenizante do Zeranol (DESCHAMPS et al., 1987b), não se manifestem (ou o façam de uma maneira subclínica) em animais tratados após a puberdade, como foi neste caso.

Porém, a ausência de alterações específicas na qualidade do sêmen dos touros tratados com DES é contrária às observações de CUPPS et al. (1960) e GUSTAFSSON (1966), embora os referidos autores tenham empregado injeçðes parenterais de estrogênio, ao invés de implentas contendo DES, conforme o presente experimento. Ainda referindo-se a estes autores, deve se considerar que, para que tais alteraçð̃es fossem observadas no sêmen, seriam nescessárias doses variando de 5 a $30 \mathrm{mg}$ por dia de estrogênio parental. Implantes de $24 \mathrm{mg}$ de DES, capazes de determinar um maior ganho de peso, em bovinos (PIMENTEL et al., 1977), devem liberar quantidades infinitamente menores de estrógeno, do que aquelas referidas na bibliografia.
Observando-se a Figura 1(a-e), pode se notar que, durante os primeiros 40 dias de experimento, houve uma melhora significativa nas diversas características do sêmen, bem como no ganho de peso, independentemente dos tratamentos executados. E sabido que, para um melhor resultado do uso de anabolizantes, em termos de ganho de peso, é fundamental, uma boa disponibilidade de alimento (CHAPMAN et al., 1964). Conforme a Figura 1a, houve um ganho de peso significativo até 100 dias após o início dos tratamentos, indicando que havia uma disponibilidade de nutrientes possivelmente satisfatoria, para que os animais implantados pudessem demonstrar um maior ganho de peso que os animais do grupo controle. Como tal não foi observado, não se pode descartar a possibilidade da ineficácia farmacologica do produto utilizado e, subseqúente ausência de alterações no quadro èspermático. $O$ emprego de motilidade, para avaliar a qualidade do sêmen, mostrou-se pouco eficaz, já que não se verificou qualquer alteração, sequer em função do tempo, conforme as demais características avaliadas.

Verifica-se, na Figura 1(b-e), um efeito significativo do dia da coleta pós-implante sobre as características do sêmen ali representadas, independentemente dos tratamentos (Grupos I, II e III). No entanto, as oscilaçðes, embora estatisticamente significativas, não fugiram dos limites de normalidade descritos por RAO (1971). Isso nos indica que tais variáveis (concentração, \% de normais, \% de anormalidades de cabeça e \% de anormalidades de cauda) são as mais representativas num exame andrológico, visando estimativas da qualidade da espermatogênese.

Convém salientar que, o presente experimento foi conduzido com apenas 3 animais compondo cada um dos grupos, o que poderia ter contribuido para uma menor capacidade de deteç̧ão das alterações esperadas. cluir que:

Com base nos presentes resultados, pode-se con-

1. DES e Zeranol, nas dosagens utilizadas neste experimento, não são capazes de afetar a qualidade do sêmen de touros adultos;

2. Os critérios utilizados para se estimar os efeitos do Zeranol e DES, sobre a qualidade do sêmen, podem não ter sido suficientemente sensíveis para detectar efeitos subclínicos de alteraçðes histologicas discretas;

3. Possivelmente o pequeno número de repeticơs, dentro das parcelas, tenha sido responsável pela não detecção de alteraçð̃es na qualidade do sêmen em decorrência dos tratamentos.

\section{FONTES DE AQUISIÇÃO}

\footnotetext{
a - Stimplant - Pfizer S.A. - Via Presidente Dutra, Km 225 C. Postal 143, Guarulhos, SP.

b - Ralgro - Tortuga - Av. Brigadeiro Faria Lima, 1409, 13/14² andares, C. Postal 20890, São Paulo, SP.
} 


\section{REFERÊNCIAS BIBLIOGRÁFICAS}

CHAPMAN, H.L., PALMER, A.Z., KIDDER, R.W. et al. Oral and implanted stilbestrol for beef cattle fattened on pasture and in drylot. Agricultural Experiment Station, Bulletin 666, University of Flórida, Gainesville, 1964.

CUPPS, P.T., LABEN, R.C., RAHLMANN, D.F. Effects of estradiol benzoate injections on the characteristics of bovine semem. J Dairy Sci, v. 43 p. $1135-1139,1960$.

CUPPS, P.T. El control endrocrino de la reproduccion en el macho, inp. Fisiologia reprodutiva del macho. Evaluacion de la fertilidad. Santiago, Chile (apostila) 13 p. 1975.

DESCHAMPS, J.C., PIMENTEL, C.A. Exame do Semen em Touros Boletim Técnico n 12. Universidade Federal de Pelotas. 29 p. 1979.

DESCHAMPS, J.C., OTTT, R.S., WESTON, P.G., et al. Effects of zeranol on reproduction in beef bulls. Luteinizing hormone and testosterone secretion in response to gonadotropin releasing hormone and human chorionic gonadotropin. Am J Vet Res, v. 48, p. 31-36. 1987 a.

DESCHAMPS, J.C., OTTT, R.S., McENTEE, K., et al. Effects of zeranol on reproduction in beef bulls. Scrotal circunference, serving ability, semen characteristics and pathological changes on the reproductive organs. Am J Vet Res, v. 48, p. 137-147, 1987 b.

GUSTAFSSON, B., Luminal contents of the bovine epididymis under conditions of reduced spermatogenesis, luminal blockage and certain sperm anormalities. Acta Vet Scand, Supplementum 17, 80 p., 1966.

JUNIEWICZ, P.E., WELSH, T.H., JOHNSON, B.H. Proc. $73^{\text {rd }}$ ASAS North Carolina, Raleigh, p. 336. 1981.
KATZENELLENBOGEN, B.S., KATZENELLENBOGEN, J.A., MORDECAI, D. Zearalenones: characterization of the estrogenic potencies and receptor interactions of a series of fungal B - resorcylic acid lactones. Endocrinology, v. 105 p. 33-40, 1979.

MATSUYAMA, S., RICHCKIND, M., CUPPS, P.T. Effects of supplemental progesterone on semen from bulls. J Dairy Sci, v. 50 p. 375-377, 1967.

PIMENTEL, S.M., ELIAS, S.J., CORDEIRO, J.M.C., et al. Efeito de anabolizantes sobre o comportamento ponderal de novilhos em campo nativo. II Encontro de Pesquisa Veterinária, Faculdade de Veterinária, Universidade Federal de Pelotas, Pelotas, p. 13, 1977.

RAO, R. Changes in the morphology of sperm during their passage through the genital tract in bulls with normal and impaired spermatogenesis. Stockholm, Sweden. 73 p. Tese Doutorado. Royal Veterinary College, Sweden. 1971.

RICHCKIND, M., MATSUYAMA, S., CUPPS, P.T. Effects of high levels of exogenous Testosterona Propionate on bovine semen. J Dairy Sci, v. 50, p. $378-380,1967$.

STEEL, R.G.D., TORRIE, J.H. Priuciples and Procedures of Statistics. Mograw-Hill Book Company, inc. New York 481 p., 1960.

STOB, M.R.S., BALDWIN, J., TUITE, F., et al. Isolation of an anbolic uterotrophic compound from corn infected with Giberella zeae. J Anim Sci, v. 196, p. 1318, 1962.

URRY, W.H., WEHRMEISTER, H.L., HODGE, E.B., HIDY, P.H. The estructure of Zearalenone. Tetrahedron Lett. p. 3109. 1966. 